\title{
A influência da torrefação na moagem do Eucalyptus in natura e torrefeito para utilização como biocombustível
}

\author{
The influence of torrefaction on the grindability of Eucalyptus in natura \\ and torrefied for use as biofuel
}

\author{
1 Erica Leonor Romão ericaromao@usp.br \\ 1 Ivo Alves Dias
}

1 Escola de Engenharia de Lorena - Universidade de São Paulo - EEL/USP.

\section{Resumo}

Neste trabalho, analisou-se a influência do processo de torrefação na moagem do Eucalyptus spp., da região do Vale do Paraíba, objetivando sua utilização como biocombustível. 0 estudo baseou-se nas reações de torrefação com temperaturas de 240,260 e $280^{\circ} \mathrm{C}$ com tempo de residência de 30 e 60 minutos, comparando as modificações ocorridas na biomassa in natura e torrificadas. Em todas as condições com biomassa torrificada, mostrou-se uma melhoria, mas nas temperaturas mais altas $\left(260\right.$ e $\left.280^{\circ} \mathrm{C}\right)$, observou-se através do MEV que a biomassa torrificada apresentava uma estrutura desarranjada, quebradiça, apresentando uma característica frágil. Essa característica também foi evidenciada na moagem, na qual $50 \%$ da biomassa torrificada de 260 e $280^{\circ} \mathrm{C}$ a 60 minutos tiveram partículas menores de $0,425 \mathrm{~mm}$, possibilitando uma padronização do biocombustível e permitindo sua utilização em condições mais exigentes quanto à estabilidade, em processos para fins energéticos, como a gaseificação.

\section{Palavras-chave}

Torrefação. Friabilidade. Biomassa. Eucalyptus. Biocombustível.

\begin{abstract}
This work studied the influence of torrefaction on the grindability of Eucalyptus spp., from the Vale do Paraiba region, aiming at its use as biofuel. The study was based on the torrefaction reactions with temperatures of 240,260 and $280^{\circ} \mathrm{C}$ with residence time of 30 and 60 minutes comparing the changes occurred in the in natura and torrefied biomass. In all conditions with torrefied biomass an improvement was observed, but in the higher temperatures (260 and $280^{\circ} \mathrm{C}$ ) it was observed through the SEM that the torrefied biomass presented a disrupted, brittle structure, presenting a fragile characteristic. This characteristic was also evidenced in milling in which $50 \%$ of the biomass torrefied at 260 and $280{ }^{\circ} \mathrm{C} 60$ minutes had particles smaller than $0.425 \mathrm{~mm}$, allowing a standardization of the biofuel and allowing its use under more demanding conditions for stability in processes for energy purposes such as gasification.
\end{abstract}

\section{Keywords}

Torrefaction. Grindability. Biomass. Eucalyptus. Biofuel.

\section{Como você deve citar?}

ROMÃO, Erica Leonor; DIAS, Ivo Alves. A influência da torrefação na moagem do Eucalyptus in natura e torrefeito para utilização como biocombustível. Cadernos UniFOA, Volta Redonda, n. 37, p. 5-13, ago. 2018. 


\section{INTRODUÇÃO}

Os biocombustíveis têm recebido significativa atenção de vários países e pesquisadores de diversas áreas, devido ao seu potencial energético, constituindo-se como uma fonte alternativa ao uso de recursos fósseis e apresentando a vantagem de ser uma fonte renovável com emissões de dióxido de carbono $\left(\mathrm{CO}_{2}\right)$ consideradas neutras, uma vez que o $\mathrm{CO}_{2}$ é absorvido durante o crescimento da planta (PORTUGAL PEREIRA, et al., 2015).

Frente a isso, entre as diversas fontes de energia, o Eucalyptus spp vem se mostrando ser promissor para geração de energia, devido, principalmente, ao grande número de espécies e também pelo fato de que a madeira corresponde a 8,7\% da matriz energética mundial e 13,9\% da brasileira (BEN, 2014).

Existem diversas tecnologias para aproveitamento da bioenergia. Dentre os processos termoquímicos, a torrefação vem se mostrando como um pré-tratamento térmico que elimina muitas limitações associadas à biomassa bruta - in natura. A torrefação não é um processo de secagem e sim uma pirólise branda da biomassa, que é realizada sob pressão atmosférica na faixa de temperatura de $200-300^{\circ} \mathrm{C}$, em ambiente inerte (MEDIC et al., 2011), objetivando obter um rico produto sólido de carbono.

Durante a torrefação da biomassa - materiais lignocelulósicos - ocorre a alteração e transformação dos três constituintes poliméricos. A hemicelulose é a componente que é mais afetada, contribuindo para a maior perda de massa, pelo fato de que suas ramificações são facilmente removidas da cadeia principal e degradam-se em baixas temperaturas. A celulose é a componente majoritária e constitui quase metade de toda sua massa seca na maioria das espécies de madeira. A lignina é a componente que apresenta menor degradação térmica, cujas reações acontecem lentamente, começando em $160^{\circ} \mathrm{C}$, até temperaturas superiores a $450^{\circ} \mathrm{C}$ (SANTOS, COLODETTE, QUEIROZ, 2013).

No trabalho realizado por Romão (2016) a partir do tronco do Eucalyptus spp da região do Vale do Paraíba, com casca, picado em cavacos de $10 \times 20 \mathrm{~mm}$, realizou-se a torrefação em reator de laboratório, sob atmosfera inerte, nas temperaturas de $240^{\circ} \mathrm{C}, 260^{\circ} \mathrm{C}$ e $280^{\circ} \mathrm{C}$, com tempo de residência de $30 \mathrm{e}$ 60 minutos. Após a reação, realizou-se o balanço de massa dos produtos, sendo: a Biomassa Torrificada (BT), água de reação e os gases não condensáveis (GNC), conforme apresentado na Figura 1.

Figura 1 - Balanço de massa das reações de torrefação com Eucalyptus spp

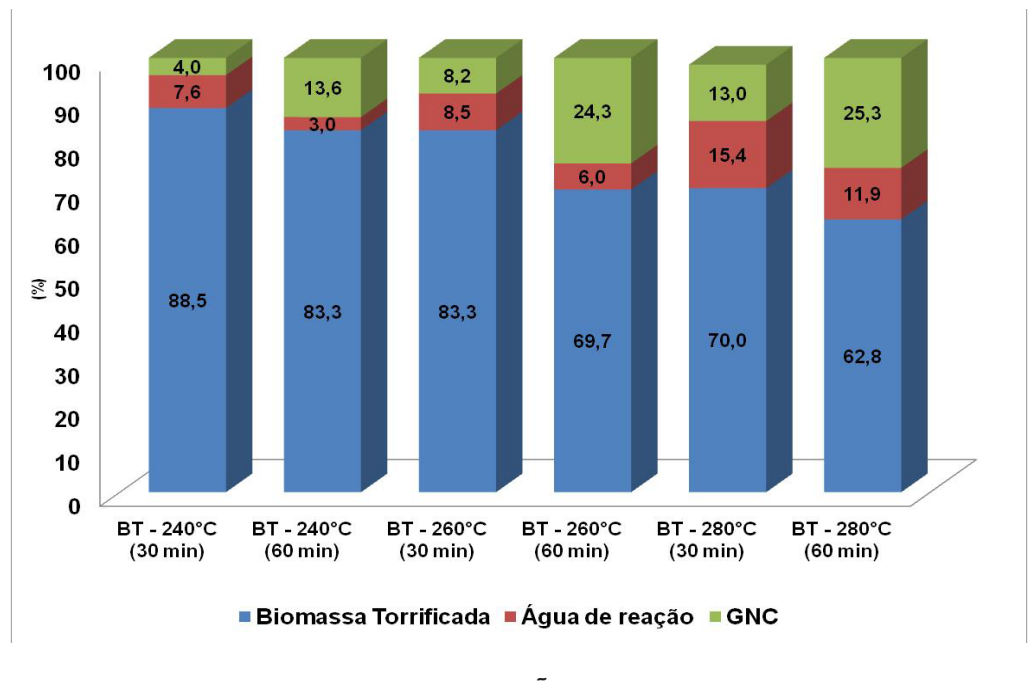

Fonte: ROMÃO, 2016. 
Observou-se que o maior rendimento gravimétrico encontrado foi no tratamento de $240^{\circ} \mathrm{C} \mathrm{com}$ 30 minutos de tempo de residência, com valores de rendimento para a biomassa torrificada de $88,5 \%$. Já o ensaio que apresentou menor rendimento gravimétrico $(62,8 \%)$ foi a $280^{\circ} \mathrm{C}$, com 60 minutos de tempo de residência. Como pode ser visto, a torrefação sofre influência de diversos fatores, tais como: a composição e granulometria da biomassa, temperatura e tempo de reação interferindo diretamente no rendimento gravimétrico dos produtos.

Essas interferências também afetam as características do produto, como pode ser vista na Figura 2, que relaciona a porcentagem do teor de carbono fixo, da conversão de biomassa torrificada e o poder calorífico superior (PCS). Pode-se observar que existe uma relação direta do aumento de temperatura com o carbono fixo e poder calorífico da biomassa.

Figura 2 - Teor de Carbono Fixo, Porcentagem de Conversão e PCS

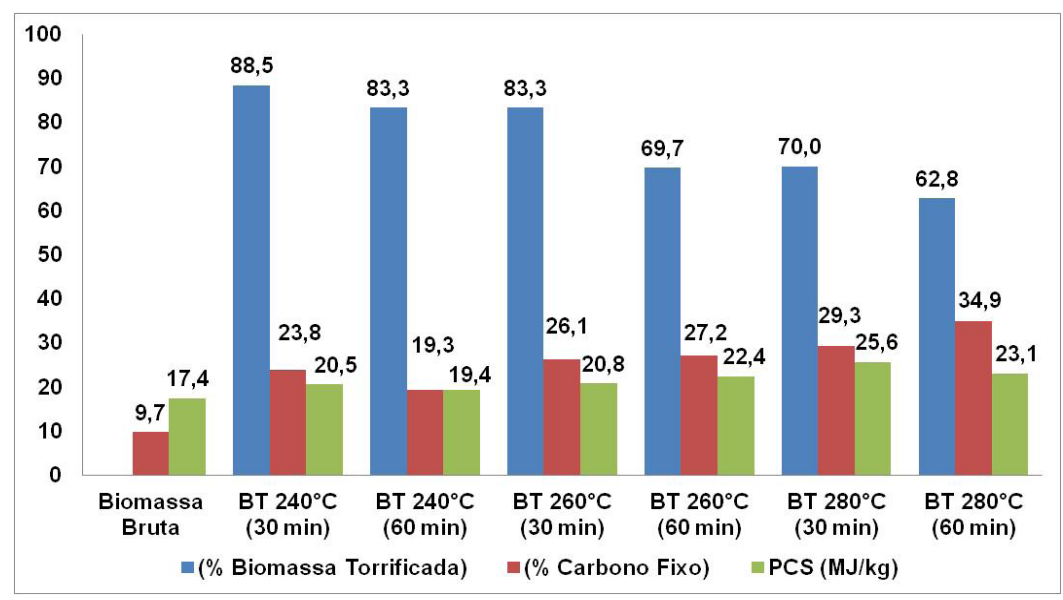

Fonte: ROMÃO, 2016.

Essas variações se devem ao fato de que há degradação dos componentes químicos majoritários da biomassa bruta, principalmente da hemicelulose, que é mais sensível à degradação térmica, em relação à celulose e a lignina. Existem dois tipos de torrefação, uma que ocorre até $240^{\circ} \mathrm{C}$ denominada de mais branda, quando ocorre maior decomposição da hemicelulose, afetando somente um pouco a celulose e lignina. E uma mais forte, acima de $270^{\circ} \mathrm{C}$, afetando também a celulose e lignina (VAN DER STELT et al. 2011), como pode ser visto pela queda na quantidade de biomassa torrificada.

A torrefação visa melhorar alguns inconvenientes da biomassa in natura, tais como: natureza fibrosa, propriedades físicas não uniformes, o baixo teor de carbono fixo associado ao alto teor de matérias voláteis, baixa densidade energética, natureza higroscópica e baixa densidade aparente. (NHUCHHEN et al., 2014; PHANPHANICH, 2011; MEDIC et al., 2011). A natureza fibrosa da biomassa bruta não só aumenta o seu custo de moagem, mas é também responsável pela inconsistência nas dimensões das partículas, aumentando as dificuldades em pulverizar um pó fino, tal como exigido para a co-combustão com carvão mineral pulverizado em caldeira (TUMULURU et al., 2011). Uma das características da madeira torrificada é o aumento da friabilidade, ou seja, mais fácil de gerar finos, mas menos friável que o carvão, assim com menos perdas na forma de finos.

Dessa forma, com vistas ao problema da natureza fibrosa da biomassa in natura, será avaliada, neste trabalho, a influência da torrefação na moagem da biomassa de Eucalyptus spp da região do Vale do Paraíba, para utilização como biocombustível nas amostras produzidas por ROMÃO (2016) no 
processo de torrefação, nas temperaturas de $240^{\circ} \mathrm{C}, 260^{\circ} \mathrm{C}$ e $280^{\circ} \mathrm{C}$ com tempo de residência de 30 e 60 minutos, visando a sua aplicação na forma pulverizada.

\section{MATERIAIS E MÉTODOS}

\subsection{Obtenção das amostras}

As amostras de Eucalyptus spp, da região do Vale do Paraíba, utilizadas neste trabalho, foram produzidas por ROMÃO (2016), recebidas com $15 \%$ de teor de unidade e torrificadas, nas temperaturas de $240^{\circ} \mathrm{C}, 260^{\circ} \mathrm{C}$ e $280^{\circ} \mathrm{C}$ com tempo de residência de 30 e 60 minutos, permanecento com umidade da ordem de $2 \%$ após o processo.

\subsection{Caracterização Microestrutural}

Realizou-se a caracterização microestrutural das amostras por micrografia eletrônica de varredura (MEV) em aparelho da marca LEO 1450 VP, com a finalidade de acompanhar as alterações ocorridas nos materiais após reações de torrefação nas três faixas de temperaturas, $240,260280^{\circ} \mathrm{C} c o m$ tempo de residência de 30 e 60 minutos. As amostras da biomassa in natura e torrificada foram depositadas, na forma de cavaco, sobre fita de carbono e metalizadas com ouro para garantir a condução dos elétrons, a fim de melhor contraste da imagem. Esse procedimento se faz necessário por se tratar de amostras de natureza orgânica.

\subsection{Etapa de moagem das amostras in natura e torrificadas}

As amostras de biomassa torrificada nas temperaturas de $240^{\circ} \mathrm{C}, 260^{\circ} \mathrm{C}$ e $280^{\circ} \mathrm{C}$, com tempo de residência de 30 e 60 minutos, e a amostra de biomassa in natura são oriundas de reações realizadas em duplicatas, as quais foram misturadas, quarteadas e moídas em moinho de facas com peneira de 10 mesh ( $2 \mathrm{~mm}$ de abertura), e as amostras de biomassa torrificada com 60 minutos de tempo de residência também foram moídas em moinho de bolas e, para efeitos de comparação, os ensaios de moagem foram realizados em duplicatas. Todas as amostras após moagem foram peneiradas em um vibrador de peneiras e separadas em quatro frações, a fim de se avaliar as variações ocorridas na granulometria, nas diferentes temperaturas.

\section{RESULTADOS E DISCUSSÃO}

\subsection{Caracterização Microestrutural}

A micrografia da estrutura da biomassa in natura de Eucalyptus spp e das amostras de biomassa torrificadas nas três faixas de temperaturas de 240,260 e $280^{\circ} \mathrm{C}$ e tempo de residência de 30 e 60 minutos foi realizada na forma de cavacos com magnitude de 500 vezes, conforme Figura 3. Como pode ser visto, as micrografias mostraram as características de espécie de madeira dura, sendo composta por:

- Elementos de vasos: Os elementos de vasos não são retos, podendo sofrer um desvio no plano tangencial ou radial e servem para transporte de água. Quanto mais elementos de vasos, mais porosa é a madeira (LEWIN; GOLDSTEIN, 1991).

- Células radiais: são células horizontais que servem para dar sustentação à árvore e compõem cerca de 10 a $20 \%$ do volume da madeira (LEWIN; GOLDSTEIN, 1991). 
- Fibras: são células longas com extremidades fechadas (pontiagudas) e servem para dar sustentação à planta. Ocupam cerca de $26 \%$ a $75 \%$ da madeira (LEWIN; GOLDSTEIN, 1991).

Observando a morfologia das micrografias da figura 3 (a) - biomassa in natura e comparando com as figuras 3 (b), (c) e (d) da biomassa torrificada, podemos evidenciar uma estrutura desarranjada, quebradiça, apresentando uma característica de material mais frágil. Nota-se que, à medida que ocorre o aumento de temperatura e o tempo de residência, essa estrutura fica mais desarranjada, as fibras ficam desorganizadas e vão perdendo a forma, sendo evidenciados estágios de degradação da biomassa. Essa característica desarranjada e escamada também foi evidenciada por Silva (2017), quando trabalhava com torrefação utilizando resíduos de coco e por Tumuluru (2017) com bagaço de cana torrificado. $\mathrm{O}$ autor comenta sobre a alteração da estrutura da fibra, devido à decomposição da hemicelulose, favorecendo o processo de moagem da biomassa torrificada, sendo essa propriedade favorável, levando a uma alta eficiência de conversão durante um processo de gaseificação. 
Figura 3 - Micrografia MEV da biomassa in natura e torrificada do Eucalyptus spp.

(a) Biomassa in natura

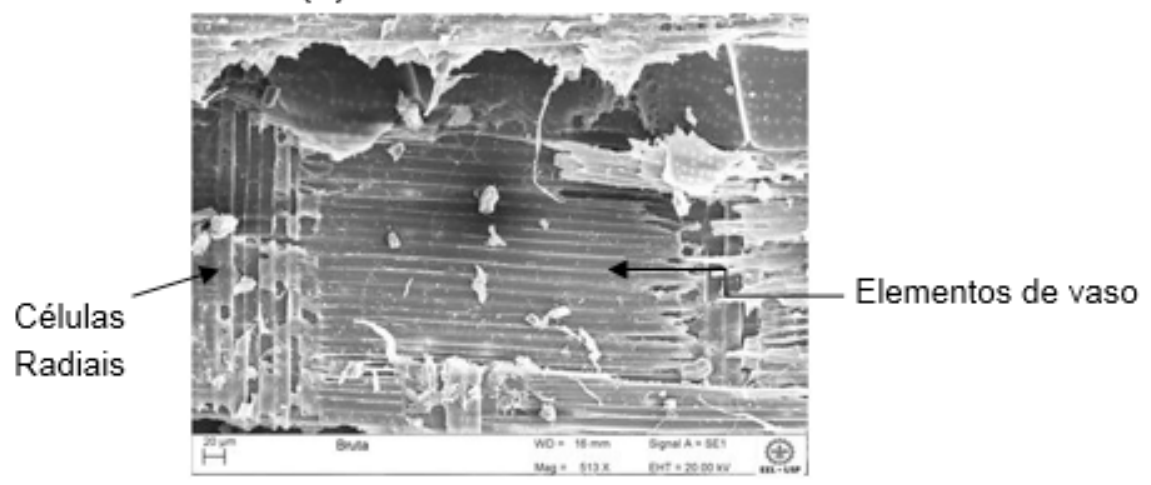

(b) $\mathrm{BT} 240^{\circ}(30 \mathrm{~min})$

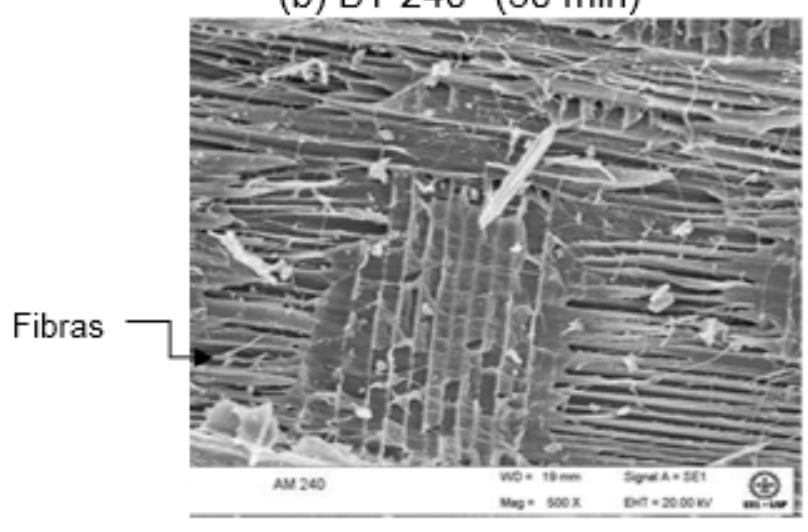

(c) BT $260^{\circ} \mathrm{C}(30 \mathrm{~min})$

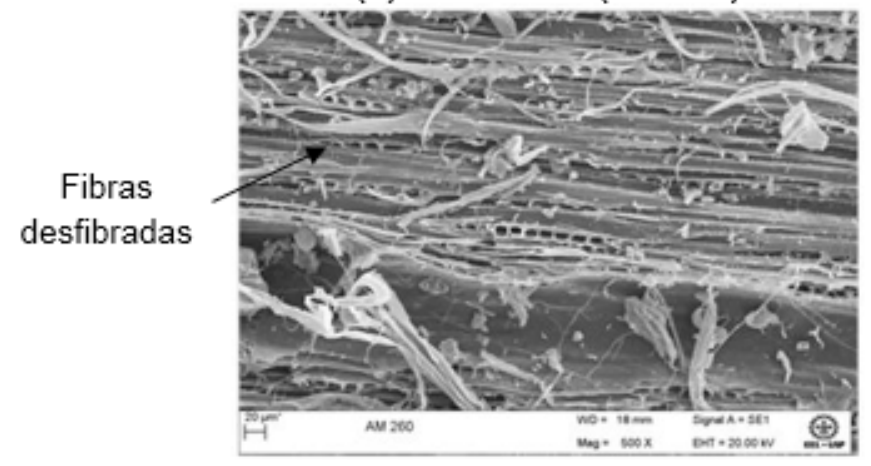

(d) $\mathrm{BT} 280^{\circ} \mathrm{C}(30 \mathrm{~min})$

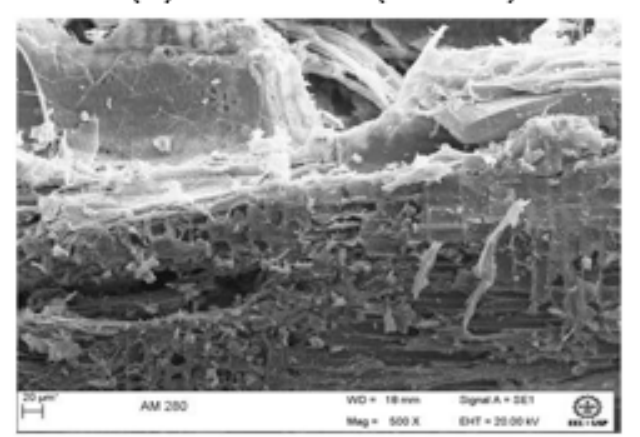

(b) BT $240^{\circ} \mathrm{C}(60 \mathrm{~min})$

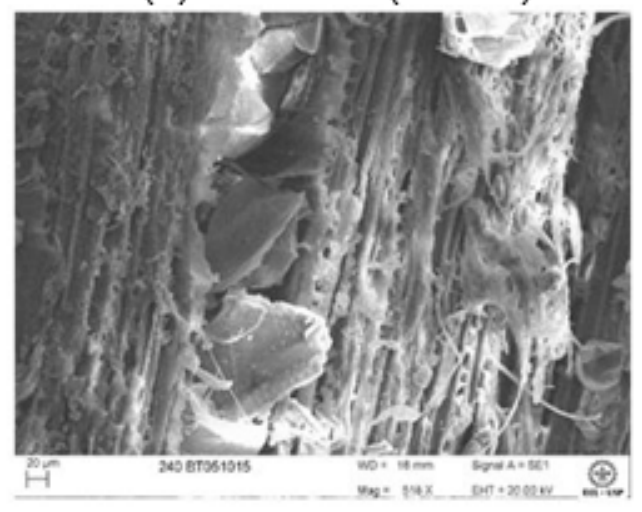

(c) $\mathrm{BT} 260^{\circ} \mathrm{C}(60 \mathrm{~min})$

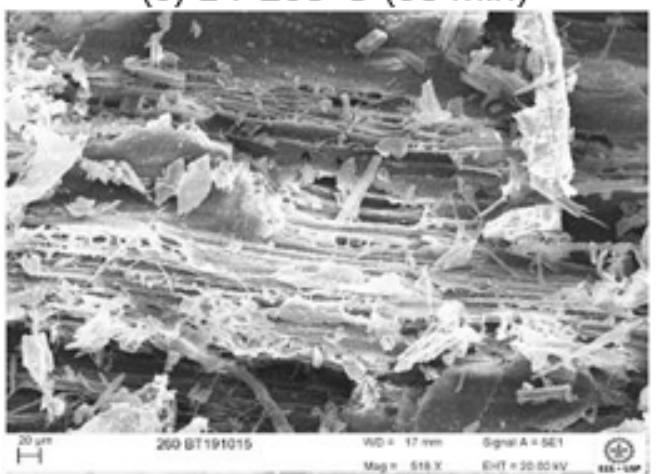

(d) $\mathrm{BT} 280^{\circ} \mathrm{C}(60 \mathrm{~min})$

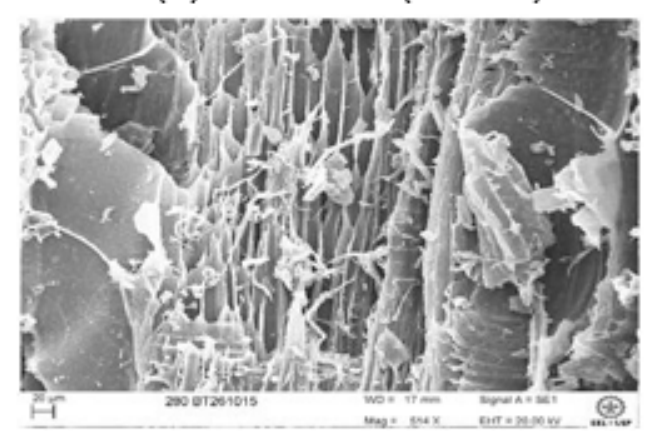

Fonte: dos autores, 2016. 


\subsection{Etapa de moagem das amostras in natura e torrificadas}

As amostras da biomassa de Eucalyptus in natura e torrificada foram moídas em moinho de facas com peneira de $2 \mathrm{~mm}$ e peneiradas em 5 frações de tamanho: >0,425; 0,425-0,212; 0,212-0,106; $0,106-0,075$ e $<0,075 \mathrm{~mm}$. 0 ensaio de moagem foi realizado em duplicata $(n=2)$ e a distribuição de partículas segue conforme apresentado na Tabela 1.

Tabela 1 - Distribuição granulométrica das amostras moídas em moinho de faca $(\% \mathrm{~m} / \mathrm{m})$

\begin{tabular}{cccccc}
\hline $\begin{array}{c}\text { abertura da peneira } \\
(\mathrm{mm})\end{array}$ & $>0,425$ & $0,425-0,212$ & $0,212-0,106$ & $0,106-0,075$ & $<0,075$ \\
\hline Biomassa in natura & $80,84 \pm 1,34$ & $11,07 \pm 0,48$ & $4,38 \pm 0,13$ & $1,25 \pm 0,14$ & $2,44 \pm 0,15$ \\
BT $240^{\circ} \mathrm{C}-30 \mathrm{~min}$ & $71,54 \pm 1,49$ & $14,88 \pm 0,35$ & $7,52 \pm 0,29$ & $2,65 \pm 0,25$ & $5,99 \pm 0,27$ \\
BT $240^{\circ} \mathrm{C}-60 \mathrm{~min}$ & $65,90 \pm 1,52$ & $17,07 \pm 0,41$ & $8,13 \pm 0,23$ & $2,89 \pm 0,24$ & $3,39 \pm 0,15$ \\
BT $260^{\circ} \mathrm{C}-30 \mathrm{~min}$ & $63,70 \pm 1,72$ & $18,41 \pm 0,47$ & $8,70 \pm 0,39$ & $3,76 \pm 0,35$ & $5,40 \pm 0,23$ \\
BT $260^{\circ} \mathrm{C}-60 \mathrm{~min}$ & $52,88 \pm 1,23$ & $21,73 \pm 0,35$ & $10,32 \pm 0,47$ & $5,92 \pm 0,53$ & $9,12 \pm 0,33$ \\
BT $280^{\circ} \mathrm{C}-30 \mathrm{~min}$ & $60,87 \pm 1,63$ & $19,73 \pm 0,21$ & $9,02 \pm 0,52$ & $4,61 \pm 0,46$ & $5,75 \pm 0,24$ \\
BT $280^{\circ} \mathrm{C}-60 \mathrm{~min}$ & $48,16 \pm 1,26$ & $23,58 \pm 0,44$ & $9,97 \pm 0,52$ & $6,50 \pm 0,35$ & $11,76 \pm 0,37$ \\
& & Fonte: dos autores, 2016. & &
\end{tabular}

Como pode ser visto, $80,84 \%$ da biomassa in natura ficou retida na peneira de $0,425 \mathrm{~mm}$. Essa dificuldade de redução do tamanho das partículas se deve à característica fibrosa da biomassa. Nas amostras de biomassa torrificada, há uma melhora nas características de moagem com o aumento da temperatura do processo de torrefação, sendo que as biomassas torrificadas a $260^{\circ} \mathrm{C}$ e $280^{\circ} \mathrm{C}$ com 60 minutos apresentaram retenção, respectivamente, de 52,88 e $48,16 \%$ na peneira de $0,425 \mathrm{~mm}$, significando uma melhoria na redução de partícula do combustível torrificado da ordem de $40 \%$ em relação a biomassa in natura. Essa redução também foi observada por Wang (2017) em madeira in natura da Noruega torrificada a $275^{\circ} \mathrm{C}$ com 60 minutos - redução na mesma ordem de grandeza $(42 \%)$. A distribuição granulométrica mostrou que quanto maior a temperatura e tempo de residência, maior a friabilidade, fragilidade da biomassa torrificada, reduzindo o esforço e consumo de energia no processo de moagem. Assim a torrefação melhora não somente o processo de moagem, mas também as condições de fluidização para utilização do biocombustível durante a gaseificação.

Já a moagem utilizando moinho de bolas apresentou distribuição granulométrica com uma maior concentração de massa nas peneiras mais finas $(n=2)$, como pode ser visto na Tabela 2 . As amostras de biomassas torrificadas a $260^{\circ}$ e $280^{\circ} \mathrm{C}$ com 60 minutos apresentaram retenção de 35,61 e $21,55 \%$, respectivamente, na peneira de $0,425 \mathrm{~mm}$. Isso se deve ao fato de que o tempo de contato da amostra no moinho de bolas ter sido superior em relação ao moinho de facas. Já na amostra de biomassa in natura, não foi possível sua moagem em moinho de bolas, no qual ela foi somente amassada e não moída. 
Tabela 2 - Distribuição granulométrica das amostras moídas em moinho de bolas (\%m/m)

\begin{tabular}{cccccc}
\hline $\begin{array}{c}\text { abertura da peneira } \\
(\mathrm{mm})\end{array}$ & $>0,425$ & $0,425-0,212$ & $0,212-0,106$ & $0,106-0,075$ & $<0,075$ \\
\hline BT $240^{\circ} \mathrm{C}-60 \min$ & $65,06 \pm 1,71$ & $7,26 \pm 0,33$ & $3,73 \pm 0,31$ & $5,19 \pm 0,38$ & $18,76 \pm 0,33$ \\
BT $260^{\circ} \mathrm{C}-60 \mathrm{~min}$ & $35,61 \pm 1,53$ & $6,87 \pm 0,27$ & $12,86 \pm 0,43$ & $22,82 \pm 0,57$ & $21,83 \pm 0,41$ \\
BT $280^{\circ} \mathrm{C}-60 \min$ & $21,55 \pm 1,21$ & $2,99 \pm 0,19$ & $6,38 \pm 0,47$ & $21,26 \pm 0,49$ & $47,92 \pm 0,50$ \\
& & & & &
\end{tabular}

\section{CONCLUSÃO}

No trabalho realizado, pode-se concluir que o processo de torrefação teve uma grande influência no efeito da moagem da biomassa de Eucalyptus torrificada. Notou-se que, para as temperaturas mais altas, a biomassa torrificada apresentou estrutura mais quebradiça e desarranjada, mostrando sua fragilidade. Isso ocorre principalmente devido à decomposição da hemicelulose que é a principal fração afetada no intervalo de temperaturas de torrefação, alterando a resistência mecânica facilitando o fracionamento e a moagem da biomassa torrificada. Também ficou evidenciado, nos ensaios de moagem nas temperaturas de 260 e $280^{\circ} \mathrm{C}$ com 60 minutos, que a biomassa torrificada teve da ordem de $50 \%$ da massa com partículas menores de $0,425 \mathrm{~mm}$, desaparecendo a estrutura fibrosa indesejada da biomassa in natura.

Assim, com o aumento da friabilidade e fragilidade da biomassa torrificada, tem-se a redução do consumo de energia no processo de moagem, possibilitando alcançar granulometrias pequenas para utilização do biocombustível, por exemplo, em ventaneiras de alto-forno. Outra vantagem também é a padronização do produto, que permite sua utilização em condições mais exigentes quanto à estabilidade, como é o caso dos processos de gaseificação. 


\section{REFERÊNCIAS}

ARIAS, B.; PEVIDA, C.; FERMOSO, J.; PLAZA, M. G.; RUBIERA, F.; PIS, J. J.; Influence of torrefaction on the grindability and reactivity of woody biomass. Fuel Processing Technology, 89 169-175, 2008.

BALANÇO ENERGÉTICO NACIONAL - BEN 2014. Disponível em: <http://www.ben.epe.gov.br>. Acesso em: 18 jan. 2016.

LEWIN, M.; GOLDSTEIN, I. S. Wood Structure and Composition.EUA: Marcel Dekker, 1991. New York: Marcel Dekker, Inc.

MEDIC, D.; DARR, M.; SHAH, A.; POTTER, B.; ZIMMERMAN, J. Effects of torrefaction process parameters on biomass feedstock upgrading. Fuel, 91 (1) 147-154, 2011.

NHUCHHEN, D. R.; BASU, P.; ARCHARYA, B. A comprehensive review on biomass torrefaction. International Journal of Renewable Energy \& Biofuels, v. 2014, 2014.

PHANPHANICH, M.; MANI, S. Impact of torrefaction on the grindability and fuel characteristics of forest biomass. Bioresource Technology 102, 1246-1253, 2011.

PORTUGAL-PEREIRA, J.; SORIA, R.; RATHMANN, R.; RSCHAEFFER; ASZKLO. "Agricultural and agroindustrial residues- to-energy in Brazil: Techno economic and environmental assessment", Biomass and Bioenergy, v. 81, 2015.

ROMÃO, E. L.; DIAS, I. A.; CONTE, R. A. e PINATTI, D. G.; Avaliação do Efeito da Torrefação de Biomassa Lenhosa Visando à produção de Biocombustível para Fins Energéticos. XXI Congresso Brasileiro de Engenharia Química - COBEQ, Fortaleza, CE, 2016.

SANTOS, F.; COLODETTE, J.; QUEIROZ, J. H.;Bioenergia e biorrefinaria Cana-de-açucar e Espécies Florestais. Viçosa: Suprema gráfica e Editora Ltda. 2013.

SILVA, A. G.; SANTOS, A. T.; BANDEIRA, M. L. S. F.; OLIVEIRA, P. F. Estudo do Processamento de Torrefação de Resíduos de Biomassa para Fins Energéticos. Revista Brasileira de Ciências Ambientais, $n^{\circ} 45$, Set, 2017.

TUMULURU, J. S.; ANUKAN, A.; MAMPHWELI, S.; OKOH. O.; REDDY. P. Influence on the conversion efficiency of the gasification process of sugarcane bagasse. Bioengineering, 4, 1-22, 2017.

Tumuluru, J. S.; Sokhansanj, S.; Hess, J. R.; Wright, C. T; Boardman, R. D. A Review on biomass torrefaction process and product properties for energy applications. Industrial Biotechnology, 7, 384-401, 2011. 